International Journal of Biomedicine | June 2021 - Volume 11, Issue Suppl_1: Abstracts from the Third Russian International Conference "Cryo-electron microscopy 2021: achievements and prospects"

ORAL ABSTRACT PRESENTATIONS

SESSION TITLE: STRUCTURE OF MEMBRANE PROTEINS

DOI: 10.21103/IJBM.11.Suppl_1.OR3

\title{
Abstract OR-3: Integrative Structural Study of the Complex of Snake Toxin WTX with a7-type Nicotinic Acetylcholine Receptor
}

\author{
Ekaterina N. Lyukmanova ${ }^{1}$, Maxim M. Zaigraev ${ }^{1}$, Dmitrii S. Kulbatskii ${ }^{1}$, \\ Milita V. Kocharovskaya $^{1}$, Yury M. Chesnokov ${ }^{2}$, Anton O. Chugunov ${ }^{1}$, \\ Mikhail P. Kirpichnikov ${ }^{1,3}$, Zakhar O. Shenkarev ${ }^{1}$ \\ ${ }^{1}$ Shemyakin-Ovchinnikov Institute of Bioorganic Chemistry, \\ Russian Academy of Sciences, Moscow, Russia \\ ${ }^{2}$ National Research Center "Kurchatov Institute," Moscow, Russia \\ ${ }^{3}$ Biological Faculty, Lomonosov Moscow State University, Moscow, Russia
}

Background: Nicotinic acetylcholine receptors are ligand-gated ion channels present in the nervous system, epithelium, and the immune system. The $\alpha 7$-type nicotinic receptor $(\alpha 7-\mathrm{nAChR})$ is a homopentameric membrane protein containing five ligand binding sites located at the interface between subunits in the extracellular domain of the receptor. $\alpha 7-n A C h R$ is considered a promising target for the treatment of cancer and cognitive dysfunction in Alzheimer's disease, schizophrenia, and depression. WTX is a non-conventional three-finger neurotoxin from the Naja kaouthia venom inhibiting $\alpha 7$-nAChR. WTX structure consists of three loops protruding from the "head" (core) stabilized by a system of disulfide bonds.

Methods: The complex of the $\alpha 7-n A C h R$ extracellular domain with a recombinant analogue of WTX was studied by cryo-electron microscopy. The structure of the complex of full-length $\alpha 7-n A C h R$ with the toxin in the membrane environment was reconstructed by in silico molecular modeling. Interaction of WTX with the lipid membrane was confirmed by NMRspectroscopy.

Results: Analysis of electronic images confirmed the homopentameric organization of the extracellular domain with a diameter of $\sim 9 \mathrm{~nm}$ and a height of $\sim 7 \mathrm{~nm}$. On the electron density map, additional regions corresponding to five WTX molecules located at the intersubunit interfaces of the domain were observed. Fitting the known spatial structures of the extracellular domain and 
the WTX toxin into the obtained electron density made it possible to reconstruct the structure of the complex (although with a low resolution of $\sim 8 \AA$ due to the predominant orientation of particles in the ice) and to determine the topology of the toxin-receptor interaction. It was revealed that WTX interacts with the extracellular domain of $\alpha 7$-nAChR by the loop II, while the loop I and the toxin's head seem to interact with the surface of the lipid membrane surrounding the receptor. Model of the complex of the full-length $\alpha 7-n A C h R$ receptor with WTX in the membrane environment corresponding to the neuronal membrane was constructed using computer simulation methods. Molecular dynamics for $>1500$ ns confirmed the stability of the complex. The predicted membraneactive site of the WTX molecule includes residues Lys13 and Arg18. The study of WTX and its mutants Lys13Ala and Arg18Ala by NMR-spectroscopy confirmed the importance of these residues for interaction with lipid membrane.
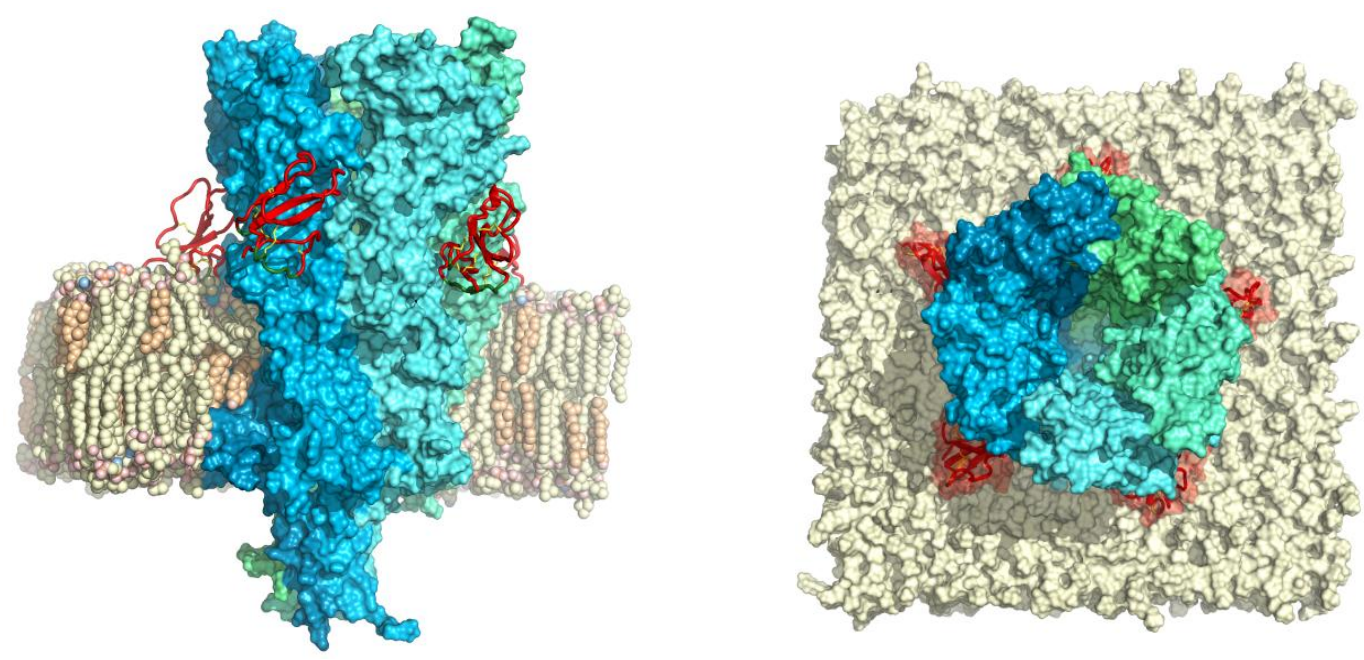

Figure 1. The model of the complex of $\alpha 7-n A C h R / W T X$ in the membrane environment. Side and top views.

Conclusion: Interaction mode of non-conventional neurotoxins with $\mathrm{nAChR}$ has been determined for the first time.

Key Words: Three-finger toxin $\bullet \alpha 7$-nAChR $\bullet$ cryo-EM $\bullet$ neuronal membrane

This work was supported by the Russian Science Foundation (project No. 1974-20163)

*Corresponding author: Ekaterina Lyukmanova. E-mail: ekaterinalyukmanova@yandex.ru

International Journal of Biomedicine. 2021;11 Suppl 1: S7-8.

doi: 10.21103/IJBM.11.Suppl_1.0R3

(C)2021 International Medical Research and Development Corporation 